\title{
Impedance Boundary Conditions for Pseudo-Spectral Time-Domain Methods in Room Acoustics
}

\author{
Carlos Spa ${ }^{\text {a,b }}$, Adan Garriga ${ }^{\mathrm{c}, \mathrm{d}}$, Jose Escolano $^{\mathrm{e}}$ \\ ${ }^{a}$ Departament de Tecnologies de la Informació $i$ les Comunicacions \\ Universitat Pompeu Fabra, Passeig de Circumval.lació 8, 08003, Barcelona, Spain.

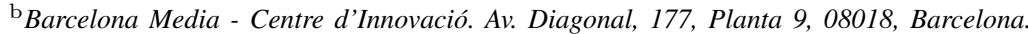 \\ ${ }^{\mathrm{c}}$ International Center for Numerical Methods in Engineering, Universidad Politécnica de Cataluña, \\ Gran Capitán s/n, 08034 Barcelona, Spain. \\ ${ }^{\mathrm{d}}$ Fundació Centre Pitiús d'Estudis Avançats. Palau de Congressos, 07840, Sta. Eulària, Ibiza, Spain.

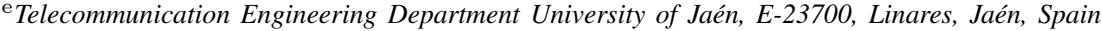 \\ e-mail: carlos.spa@upf.edu, agarriga@cimne.upc.edu, escolano@ujaen.es
}

\begin{abstract}
The Pseudo-Spectral Time Domain (PSTD) method is an alternative time-marching method to classical leapfrog finite difference schemes in the simulation of wave-like propagating phenomena. It is based on the fundamentals of the Fourier transform to compute the spatial derivatives of hyperbolic differential equations. Therefore, it results in an isotropic operator that can be implemented in an efficient way for room acoustics simulations. However, one of the first issues to be solved consists on modeling wall absorption. Unfortunately, there are no references in the technical literature concerning to that problem. In this paper, assuming real and constant locally reacting impedances, several proposals to overcome this problem are presented, validated and compared to analytical solutions in different scenarios.
\end{abstract}

Key words: PSTD method, Impedance Boundary Conditions, FDTD method

\section{Introduction}

The sound distribution in a room is the consequence of the complex sound wave phenomena appearing due to the geometry of the room and the absorbing properties of the walls, ceiling and floor [1]. Furthermore, the particular combination of the acoustic phenomena, i.e. corners diffraction, frequencydependent absorption.. gives a distribution of acoustic variables which strongly depends on position and time.

The acoustic phenomena in rooms is very complex and in general it is extremely hard to find analytical expressions for the complete characterization of the acoustic field. For this reason, the use of computers to predict the acoustic field in rooms has become an important tool for the acoustic design in room acoustics [2].

Usually, two big groups are considered to classify computer room acoustics simulations [3]: geometric methods, and wavebased methods. The first group embraces a set of algorithms based on the assumption that sound wavelengths are significantly smaller than the size of the objects found in the room. Among these methods, the most popular ones are the raytracing based algorithms [4], the image-source method [5] and the recently proposed beam tracing methods [6]. Nevertheless, a considerable disadvantage of these methods is the fact that they do not include directly wave phenomena such as interferences or diffraction. Therefore, the results obtained with such algorithms are inaccurate in the low frequency range, leading to erroneous predictions in that range.

For that reason, the second group of wave-based methods arises as a very interesting alternative. These methods are based on numerical solutions of the partial differential equations that govern the sound propagation in rooms. A room is characterized by its geometry and by the materials of the walls, ceiling and floor, which are the boundary conditions of the system. Since to analytically solve a boundary value problem is not straightforward, wave-based methods are the best option to found a solution with a high degree of accuracy. From that point of view, a considerable number of alternatives are commonly applied: from the Finite Element Methods [7,8] or Boundary Element Methods [9] which were developed originally for solving acoustic problems in the frequency domain; to the Finite Difference in the Time Domain (FDTD) [10] or Digital Waveguide Mesh methods (DWM) [11] usually devised to solve transient acoustic phenomena. 
All these wave methods provide different advantages and disadvantages depending on their computational cost and complexity. However, the most prominent difference lies in their applicability: the frequency-based methods provide results for a particular frequency, and thus, for steady-state situations; whereas if the scope of the simulation is to predict the impulse responses of an enclosure, the time-domain methods need just one simulation to obtain the results. For that reason, this last group of simulation methods is widely used in room acoustics, where the computation of impulse responses is a central issue. It is worth mentioning that in the last years variants of FEM and BEM have been developed to treat acoustic problems in the time-domain Ref. [12].

The most used time-domain (or discrete-time) methods in room acoustics are the previously mentioned FDTD and DWM, together with their more sophisticated variants such as the interpolated [13] and the triangular/tethraedrical [14,15] DWM. Recently other alternative methods such as the Transmission Line Matrix (TLM) [16] and Functional Transformation Method (FTM) [17] have been proposed.

An alternative to the common time-domain methods are the emerging class of spectral algorithms known as PseudoSpectral Time Domain (PSTD) methods. The most popular PSTD method is the so-called Fourier spectral method [18] which uses Fourier transforms for computing the spatial derivatives. Due to the fact that spatial derivatives are evaluated in a Fourier basis, the Fast Fourier Transform algorithm [19] can be used for the efficient computation of the derivatives. A major further advantage over the FDTD method is the low numerical dispersion that is observed [18,20,21]. The numerical accuracy of the Fourier spectral method for the determination of phase properties in a given structure is therefore improved with respect to the common FDTD methods. In the last years PSTD have been used in different fields such as the propagation of acoustic waves [22], modeling of piezoelectric transducers [23] or simulation of photonic devices [24].

In this paper we present a frequency independent impedance boundary condition for PSTD. These boundary conditions are appropriate for room acoustics, where the locally reacting impedance behavior has to be simulated. The results obtained from this new boundary condition algorithm are tested and validated by means of a multidimensional experiments. The issue of impedance boundary conditions for PSTD has never been treated in the technical literature and opens the possibility of using pseudo-spectral techniques in room acoustics and other related fields of research.

The paper is organized as follows: in Sec. 2 we introduce spectral methods and the explicit PSTD based on Fourier transforms is presented for the wave equation and it is compared with the FDTD method; in Sec. 3 different impedance boundary conditions are presented and tested; in Sec. 4 numerical results are discussed; and, finally, in Sec. 5 we present the conclusions. Technical aspects regarding the stability of the proposed algorithms are left to the Appendices.

\section{The PSTD algorithm}

Although the Fourier spectral PSTD method introduced by Liu [18] was originally formulated for the numerical solution of Maxwell's equations, it can be easily adapted to acoustic wave propagation [22]. In this section we review the basic formulation of the PSTD method and give a brief overview of the main features of PSTD when compared with FDTD/DWM methods.

\subsection{Formulation}

The difference between the common FDTD methods and PSTD lies in the treatment of the spatial derivatives which in the case of PSTD are computed by using discrete Fourier transforms. To illustrate this, let us consider a one dimensional domain of size $L$ and an evolving physical quantity $u(x, t)$. Consider that $\Delta x=L / N_{x}$ is used as the cell size and $N_{x}$ is the number of grid points. It is well known that the approximation of the spatial derivative can be written in general as:

$$
\frac{\partial u(x, t)}{\partial x} \approx \mathcal{F}_{x}^{-1}\left(\iota k_{x} \mathcal{F}_{x}(u(x, t))\right),
$$

where $\mathcal{F}_{x}$ and $\mathcal{F}_{x}^{-1}$ denote the Fourier transform over the $x$ axis and its inverse respectively; $k_{x}$ is the wavenumber and $\iota=$ $\sqrt{-1}$. More concretely, using the discrete Fourier transform, the spatial derivative at the locations $x=i \Delta x, i=0,1, \ldots, N_{x}-1$, is given by a trigonometric polinomial:

$$
\left(\frac{\partial u(x, t)}{\partial x}\right)_{x=i \Delta x}=\frac{1}{L} \sum_{m=-N_{x} / 2}^{N_{x} / 2-1} \iota k_{m} \widetilde{u}(m) e^{\iota k_{m} i \Delta x}
$$

where $k_{m}=2 \pi m / L$, and $\widetilde{u}(m)$ is the Fourier series

$$
\widetilde{u}(m)=\Delta x \sum_{i=0}^{N_{x}-1} u(i \Delta x) e^{-\iota k_{m} i \Delta x} .
$$

Interestingly, the discrete Fourier transforms in Eqs. (2) and (3) can be obtained efficiently by using a FFT algorithm [19], with a number of operations of the order of $\left(N_{x} \log _{2} N_{x}\right)$. It is worth mentioning the fact that from the Nyquist sampling theorem, the derivative in Eq. (2) is exact for $\Delta x \leq \lambda / 2(\lambda$ being the wavelength) ${ }^{1}$ which implies that even with two cells per wavelenght, the PSTD method does not produce phase error due to the spatial discretization [18]. This makes the PSTD method far better than the more common FDTD methods for the numerical study of high-frequency problems or if long-time solutions are needed.

An explicit form of the PSTD method for the wave equation in three dimensions can be easily derived,

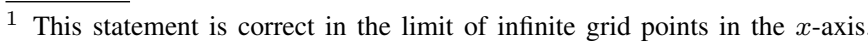




$$
\begin{aligned}
\left.p\right|_{i, j, k} ^{n+1} & =-\left.p\right|_{i, j, k} ^{n-1}+\left.2 p\right|_{i, j, k} ^{n} \\
& +(c \Delta t)^{2}\left(\tilde{\mathcal{F}}_{x}^{-1}\left[\left(-\frac{2 \pi n_{x}}{N_{x} \Delta x}\right)^{2} \tilde{\mathcal{F}}_{x}\left[\left.p\right|_{:, j, k} ^{n}\right]\right]\right. \\
& +\tilde{\mathcal{F}}_{y}^{-1}\left[\left(-\frac{2 \pi n_{y}}{N_{y} \Delta y}\right)^{2} \tilde{\mathcal{F}}_{y}\left[\left.p\right|_{i,:, k} ^{n}\right]\right] \\
& \left.+\tilde{\mathcal{F}}_{z}^{-1}\left[\left(-\frac{2 \pi n_{z}}{N_{z} \Delta z}\right)^{2} \tilde{\mathcal{F}}_{z}\left[\left.p\right|_{i, j,:} ^{n}\right]\right]\right),
\end{aligned}
$$

where $\tilde{\mathcal{F}}_{\mu}$ denote the discrete Fourier transform over the $\mu$-axis. $n_{\mu}$ is the index of the discrete Fourier Transform; $N_{\mu}$ are the total grid point over the $\mu$-axis; and the : symbol denotes all spatial samples along the $\mu$-coordinate.

The second order temporal derivative is approximated by a centered finite difference operator and the second order spatial derivative uses the Fourier techniques explained above. In Eq. (4), $c$ is the speed of sound; $p$ stands for the acoustic pressure; the index $n$ is the time step; $\Delta t$ is the time discretization; $(i, j, k)$ are the three-dimensional spatial coordinates and $\Delta x$, $\Delta y$ and $\Delta z$ represent the spatial discretization in each axis.

A standard Von Neumann analysis [26] yields the following relation for the stability Courant number, $S$, when $\Delta x=\Delta y=$ $\Delta z=\Delta$ :

$$
S=c \frac{\Delta t}{\Delta} \leq \frac{2}{\pi \sqrt{D}},
$$

where $D$ represents the dimension (in the case of Eq. (4), $D=$ 3 ). Note that $S$ in PSTD method is smaller than in FDTD method $(S \leq 1 / \sqrt{D})$ which means that PSTD is stable for larger cell sizes than FDTD (with the same time discretization step). More relevant is the fact that, from a dispersion error point of view, PSTD methods exhibit a perfectly isotropic relation, which means that the ratio between the theoretic speed of sound and the actual speed of plane waves traveling through the numerical domain, $c_{\text {num }}$, does not depend on the propagation angle $\theta[21]$.

\subsection{Additional Remarks}

One of the most important drawbacks of the FDTD methods for room acoustics applications is the inherent dispersion error $[20,26]$. As an example, we consider the dispersion relation for the classical leapfrog scheme in two dimensions [27]:

$$
\begin{aligned}
S^{-2} \sin ^{2}\left(\frac{\pi S}{N_{\lambda}}\right)= & \sin ^{2}\left(\frac{\pi c}{N_{\lambda} c_{\text {num }}} \cos \theta\right) \\
& +\sin ^{2}\left(\frac{\pi c}{N_{\lambda} c_{\text {num }}} \sin \theta\right)
\end{aligned}
$$

where $N_{\lambda}=\lambda / \Delta x$ is the number of cells per wavelength (cpw), being $\lambda$ the wavelength. From Eq. (6) it can be seen that the numerical speed of sound, $c_{\text {num }}$, depends strongly not only on the Courant stability number, $S$, and the number of cells per wavelenght, $N_{\lambda}$, but also on the direction of wave propagation across the two dimensional domain, $\theta$.
One remarkable property of the PSTD method is the isotropy of the numerical speed of propagation. More specifically, for large enough numerical domains a simple dispersion relation can be obtained [20]:

$$
\frac{c_{\mathrm{num}}}{c}=\frac{\pi}{N_{T} \sin \left(\frac{\pi}{N_{T}}\right)}, \quad N_{T}:=\frac{T}{\Delta t},
$$

where $T$ is the period of the wave and $N_{T}$ controls the time discretization. Note that the dispersion error does not depend on the angle of propagation. This fact allows to easily correct the dispersion error (at each frequency) avoiding erroneous results in the computation of acoustic impulse responses.

In the last years, some research has been devoted to the improvement of dispersion errors in FDTD/DWM methods [13,14,28]. These algorithms improve the isotropy of the numerical sound propagation at the expense of computational cost.

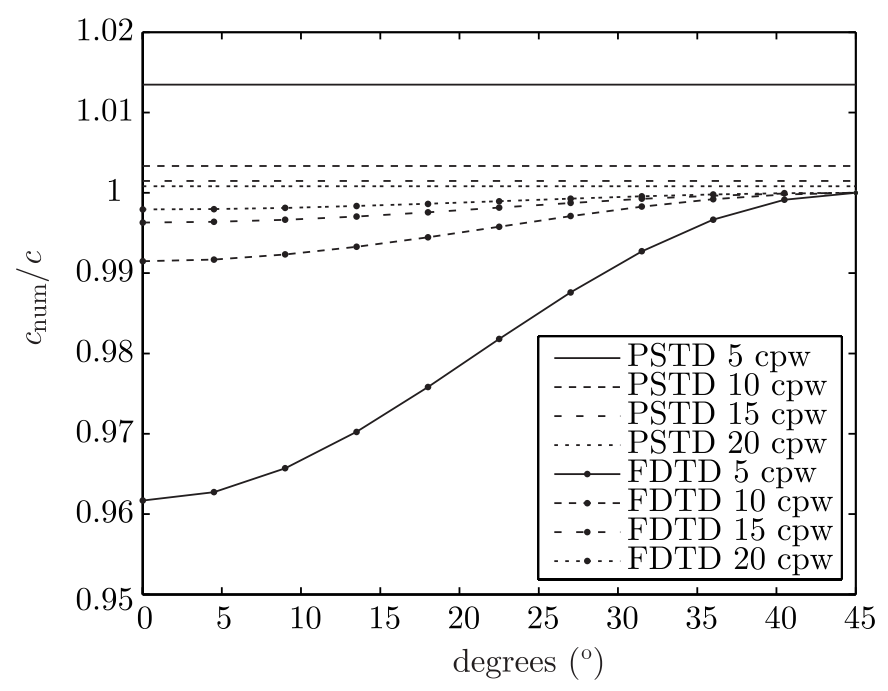

Fig. 1. Dispersion Error for the PSTD algorithm in two dimensions with $S=2 /(\pi \sqrt{2})$ and $N_{\lambda}=5,10,15,20 \mathrm{cpw}$ and for the classical leapfrog method with $S=1 / \sqrt{2}$ and $N_{\lambda}=5,10,15,20 \mathrm{cpw}$.

Figure 1 plots the normalized numerical speed of sound coming from the dispersion relations, Eq. (6) for FDTD and Eq. (7) for PSTD, as a function of the angle of propagation (with respect the $x$-axis). We use the maximum value of the Courant number, $S$, allowed for each algorithm at different values of $N_{\lambda}$. Observe that the dispersion error in the leapfrog FDTD scheme depends strongly on the angle of propagation instead of PSTD method. Moreover it is worth mentioning that PSTD gives less severe values of $c_{\text {num }}$ at each $N_{\lambda}$. Therefore, from the dispersion error point of view, the PSTD method is clearly more suitable for room acoustic applications.

Another important feature of the PSTD method in comparison with FDTD methods is the reduction in computational cost. On the one hand, PSTD simulations require the computation of Fourier transforms which can be computed very efficiently through the Fast Fourier Transform algorithms [19]; on the other hand - and probably the most compelling feature - PSTD do not produce phase error even for two cells per wavelength, i.e $N_{\lambda}=2$ [18]. This last property allows for a simulation of 
large spaces and computation of very long acoustic impulse responses. However, as can be seen in Fig. 1, $5 \mathrm{cpw}$ will still be required if the dispersion error is to be kept to about $1 \%$ or less.

\section{Boundary conditions}

In this section we present a locally reacting impedance boundary condition for the PSTD algorithm. In the past, most efforts have been mainly focused on the implementation of absorbing boundary conditions in order to avoid the Gibb's phenomenon produced by using the discrete Fourier transforms for the derivatives. The Gibb's phenomenon is produced due to the periodic boundary conditions introduced when using the discrete Fourier transform. The consequence is that the Fourier series are truncated and spurious reflection signals appear at the boundaries [29]. The best way to implement perfectly absorbing boundary conditions is the so-called Perfectly Matched Layer (PML) [30-32].

Some applications, such as room acoustics modeling and aeroacoustics, require to define partially absorbing boundary conditions. The performance of these reflecting boundary conditions in PSTD methods has been treated in very few publications [33], since to define it as a problem with different domains causes serious difficulties in their implementation [34].

\subsection{Impedance Boundary Conditions}

In this section we will give a short overview of the definition of an impedance boundary condition: specific boundary impedance $Z$ is defined for a given plane wave, as the ratio of sound pressure complex amplitude and the normal component of the associated particle velocity, $\mathbf{v}$. In the particular case of a plane wave traveling in the air, the specific acoustic impedance of that medium is $Z_{\text {air }}=\rho c$, where $\rho$ is the air density and $c$ is the speed of sound.

A plane surface with a specific boundary impedance presents a particular response depending on the shape of the incident wave. However, in some scenarios, such as room acoustics, it is reasonable to assume that there is a local linear relation between the normal component of particle velocity and the pressure at a particular point of the surface and then, a material surface may be characterized in terms of a unique specific boundary impedance. Although real scenarios would consider a frequency dependent impedance, this paper is only focused on constant, or frequency independent, impedances.

To be more concrete let us define a two dimensional domain $V$. The acoustic pressure within the region is governed by the wave equation, except for those positions, $\mathbf{y}$, which are located at the boundary, $\partial V$ (see Fig. 2). The time-domain relation between the acoustic pressure and velocity at $\partial V$ is given by

$$
p(\mathbf{x}, t)=Z \mathbf{v}(\mathbf{x}, t) \cdot \hat{\mathbf{n}},
$$

where $\hat{\mathbf{n}}$ is the normalized outward normal vector of the boundary [1], and $Z$ is a real positive constant. If a plane wave which is traveling toward a locally reacting impedance surface strikes over it with an incident angle $\theta$, a ratio between the reflected

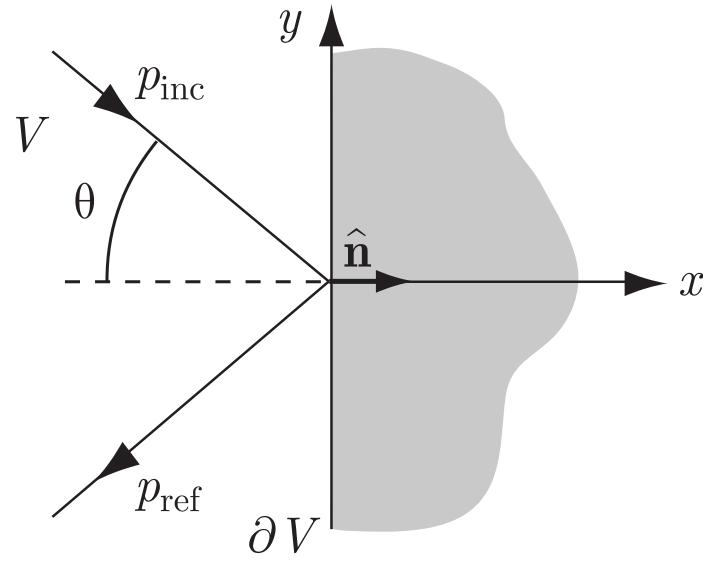

Fig. 2. Reflection from a plane surface, $\partial V$, in a two dimensional domain $V$. $\mathbf{n}$ is the normal vector associated to the boundary.

and the incident pressure, $p_{\text {ref }}$ and $p_{\text {inc }}$ respectively, is defined. This ratio is known as reflection factor $R_{\text {th }}$ and it is related to the local reacting impedance $Z$ through [35]:

$$
R_{\mathrm{th}}(\theta)=\frac{Z \cos \theta-\rho c}{Z \cos \theta+\rho c} .
$$

where $\theta$ is the angle represented in Fig. 2. Furthermore, it is assumed that $\mathbf{v}(\mathbf{x}, t)$ fulfils the linear conservation mass equation,

$$
\frac{\partial \mathbf{v}(\mathbf{x}, t) \cdot \hat{\mathbf{n}}}{\partial t}=-\frac{1}{\rho}(\nabla \cdot \hat{\mathbf{n}}) p(\mathbf{x}, t) .
$$

where $\rho$ represents the air density and $\nabla$ is the gradient vector in Cartesian coordinates. If Eq. (8) is introduced in the linear conservation mass equation, an expression for the locally reacting impedance boundary condition arises:

$$
\frac{\partial p(\mathbf{x}, t)}{\partial t}=-\frac{Z}{\rho} \frac{\partial p(\mathbf{x}, t)}{\partial \hat{\mathbf{n}}}
$$

\subsection{Numerical implementations}

In the present section, several proposals are made in order to include the impedance boundary condition in the PSTD scheme for room acoustics. For that purpose, the first step consists on discretizing the boundary condition, Eq. (11), with a uniform spatial sampling frequency $\Delta$. Without loss of generality, from now on we shall consider that the boundary is located parallel to the $y$-axis on the right hand side of a two-dimensional domain, as illustrated in Fig. 2.

In order to implement an impedance boundary condition, one may think about using a PSTD-based algorithm to perform the spatial derivatives on Eq. (11), resulting on the following scheme for those nodes $(i, j)$ located at the boundaries:

$$
\left.p\right|_{i, j} ^{n+1}=\left.p\right|_{i, j} ^{n}-\frac{Z \Delta t}{\rho} \tilde{\mathcal{F}}_{x}^{-1}\left\{\iota k_{x} \tilde{\mathcal{F}}_{x}\left\{\left.p\right|_{:, j} ^{n}\right\}\right\} .
$$

The first step after the proposal of a finite difference scheme, is to analyze the stability of the finite difference itself. Then, a Von Neumann analysis of the stability is performed [36], which 
consists on assuming a planar wave, $p(\mathbf{x}, t)=\xi^{t / \Delta t} e^{-\iota \mathbf{k}_{0}^{T} \mathbf{x}}$, and to find under which conditions $\|\xi\| \leq 1$ occurs. $\mathbf{k}_{0}$ is the wavenumber vector of the plane wave and ${ }^{T}$ indicates transposition. For Eq. (12), this analysis indicates that $Z$ has to be purely imaginary in order to keep stable that scheme (see the Appendix for details). This result indicates that this scheme is unconditionally unstable, since in the time domain, this will result into a non physic impedance boundary condition $(Z(\omega) \neq$ $Z^{*}(-\omega)$, see Ref. [38] for details).

In order to overcome this handicap, an alternative form of Eq. (11) is proposed, which consists on applying a time derivative at both sides of Eq. (12),

$$
\frac{\partial^{2} p(\mathbf{x}, t)}{\partial t^{2}}=-\frac{Z}{\rho} \frac{\partial^{2} p(\mathbf{x}, t)}{\partial t \partial x} .
$$

This new equation does not essentially change Eq. (11), but the finite differences schemes derived from this equation could differ substantially. According to the PSTD algorithm, we obtain a new scheme for the impedance boundary condition,

$$
\begin{aligned}
\left.p\right|_{i, j} ^{n+1} & =\left.2 p\right|_{i, j} ^{n}-\left.p\right|_{i, j} ^{n-1} \\
& -\frac{Z \Delta t}{2 \rho} \tilde{\mathcal{F}}_{x}^{-1}\left\{\iota k_{x} \tilde{\mathcal{F}}_{x}\left\{\left.p\right|_{:, j} ^{n+1}-\left.p\right|_{:, j} ^{n-1}\right\}\right\} .
\end{aligned}
$$

From now on, the former PSTD boundary condition (Eq. (12)) will be named as PSO1 (Pseudo-Spectral Order 1), whereas the latter (Eq. (14)), will be referred as PSO2 (Pseudo-Spectral Order 2). The results from the Von Neumann analysis on PSO2 show that it is an unconditionally stable scheme (see the Appendix for details).

Despite Eq. (14) seems to be an appropriate solution, let us propose a local use of a finite difference time domain scheme at the boundaries. Although the number of hybrid methods involving a PSTD algorithm is really small [37], it will be shown later how a hybrid solution gives fairly better results than a purely PSTD method.

A first approach consists on a central finite difference operator for the time derivatives whereas the spatial derivative uses a forward/backward finite difference operator, depending on the orientation of $\hat{\mathbf{n}}$. As before, we shall consider that the boundary is located parallel to the $y$-axis. The scheme for those nodes $(i, j)$ located at the boundary is given by:

$$
\left.p\right|_{i, j} ^{n+1}=\left.p\right|_{i, j} ^{n}-\frac{Z \Delta t}{\rho \Delta}\left(\left.p\right|_{i, j} ^{n}-\left.p\right|_{i-1, j} ^{n}\right) .
$$

Unfortunately, the previous scheme (FDO1 from now on) is stable if $Z \leq \rho c S^{-1}$, where $S=c \Delta t / \Delta$ (see the Appendix), which is a small range of impedance values. In order to overcome this handicap we follow the same procedure as before by using Eq. (13). The finite differences scheme reads:

$$
\begin{aligned}
\left.p\right|_{i, j} ^{n+1} & =\left.\frac{2 \rho \Delta}{\rho \Delta+0.5 Z \Delta t} p\right|_{i, j} ^{n}-\left.\frac{\rho \Delta-0.5 Z \Delta t}{\rho \Delta+0.5 Z \Delta t} p\right|_{i, j} ^{n-1}+ \\
& +\frac{0.5 \Delta t Z}{\rho \Delta+0.5 Z \Delta t}\left(\left.p\right|_{i-1, j} ^{n+1}-\left.p\right|_{i-1, j} ^{n-1}\right)
\end{aligned}
$$

where the time derivatives are approximated by the common central finite differences operators while the spatial derivative is approximated following the same strategy as in Eq. (15).
The Von Neumann analysis over this particular difference scheme (from now on, FDO2) demonstrates that it is unconditionally stable for all values of $Z$ (see the Appendix for a detailed derivation).

Although PSO2 and FDO2 seem to be both appropriate as candidates to be used in numerical simulations, a basic situation is simulated in order to confirm the suitability of all the previous presented schemes. The experimental system consists on a 1D scenario of 1024 cells where the input signal is located at a relative distance of 50 cells of the impedance boundary condition. In this simple experiment, the input signal is an acoustic impulse approximated by,

$$
p_{\text {source }}^{n}=\frac{\sin \left(2 \pi f\left(n-n_{0}\right) \Delta t\right)}{2 \pi f\left(n-n_{0}\right) \Delta t} \quad n \leq 36 .
$$

Note that this function has a flat frequency spectrum from 0 to $f$. All the cases are tested with $f=2500 \mathrm{~Hz}, n_{0}=$ $19, \Delta t=1 / 16000 \mathrm{~s}$ and the maximum allowed value of the Courant stability number, given by Eq. (5). This means that at the highest frequency $(f=2500)$ a minimum value of the number of cells per wavelength is obtained: $N_{\lambda} \simeq 3 \mathrm{cpw}$. The simulation is run during a convenient number of time steps in order to avoid spurious numerical reflections and to minimize the truncation error in the analysis of the spectra.

Since $Z \in[0,+\infty[$, it is more convenient to express the different boundary conditions by using as a parameter the theoretic reflection factor, $R_{\mathrm{th}} \in[-1,1]$, which comes from Eq. (9). The simulations have been carried out for values of $R_{\mathrm{th}}=$ $-1,-0.9, \ldots, 1\left(\Delta R_{\mathrm{th}}=0.1\right)$. Therefore, we compute the average error, $\epsilon$, of the numerical measured reflection factor $R_{\text {meas }}$ (measured with the one dimensional version of the experiment detailed in Section 4) with respect to the theoretic reflection factor $R_{\mathrm{th}}$, expressed in $\mathrm{dB}$ :

$$
\epsilon=20 \log _{10}\left\|R_{\mathrm{th}}-R_{\text {meas }}\right\|
$$

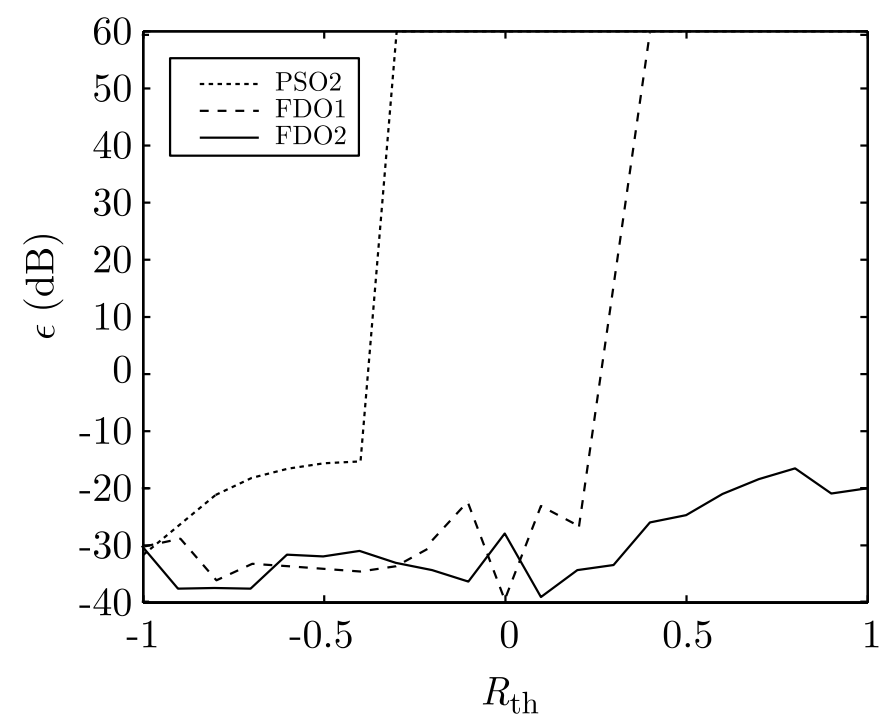

Fig. 3. Average error, $\epsilon$, between the measured numerical reflection factor, $R_{\text {meas }}$, and the theoretical one $R_{\text {th }}$ in $\mathrm{dB}$. 
Fig. 3 shows the results of that simulation. As expected from the previous analysis, the PSO1 is unconditionally unstable since $Z$ has to be purely imaginary. Surprisingly, despite PSO2 is an unconditionally stable scheme, results are stable from $R_{\mathrm{th}}=-1$ until $R_{\mathrm{th}}=-0.6(Z=0.25 \rho c)$. As it is reported in the technical literature, the reason is the fact that pseudospectral methods are very sensitive to changes in the density of the acoustic medium [34]. This means that the incorporation of impedance boundary conditions produce serious instabilities in the present case.

Fortunately, the finite differences approach give considerable better results. The results of FDO1 (see Fig. 3) are in accordance with the Von Neumann analysis. Since the scenario is a 1D simulation, the Courant number is $S=2 / \pi$ implying that the simulation should be stable until $Z \leq 1.57 \rho c\left(R_{\mathrm{th}}=0.22\right)$, which is confirmed by the numerical simulations where a divergence is found at $R_{\mathrm{th}} \approx 0.2$. However, by using FDO2 the results are completely stable for all values of $Z$ (and hence for all $\left.R_{\mathrm{th}}\right)$. This option is clearly the best one since is the unique proposed method which is unconditionally stable and the error is below $-30 \mathrm{~dB}$ from $R_{\mathrm{th}}=-1$ to $R_{\mathrm{th}}=0.5(Z \in[0,3 \rho c])$, and around $-20 \mathrm{~dB}$ for the rest of values. Furthermore, it is also noticeable that for those values of $\mathrm{Z}$ for which all the schemes are stable, the error is lower using finite-difference schemes at the boundaries than using a purely PSTD approach.

Then, as a first conclusion, the use of a finite-difference scheme FDO2, Eq. (16), for boundary conditions in PSTD schemes provide an unconditionally stable scheme which results have demonstrated good accuracy.

\section{Numerical Experiments in multidimensional scenarios}

In Sec. 3 we have shown that the boundary conditions labeled as FDO2, Eq. (16), are the most stable and accurate conditions for room acoustics applications. In this section we show not only the accuracy, but also the locally reacting behavior of FDO2 by means of proper multidimensional experiments.

\subsection{Experimental Setup}

In this section we define the experimental setup in order to test the accuracy of the FDO2 conditions as a locally reacting boundary conditions when combined with PSTD. The experimental setup used is inspired on Refs. [39,40]. The system consists on a two dimensional rectangular interpolated mesh with a sound source located at $\mathbf{x}_{s}$. Many receivers, $\mathbf{x}_{\tau_{\xi}}$ and $\mathbf{x}_{\tau_{\xi}^{\prime}}$, where $\xi=1,2,3 \ldots$, are placed along the parallel lines $\tau$ and $\tau^{\prime}$ as it is shown in Fig. 4.

Within the experimental setup of Fig. 4, two simulations are carried out: a first simulation in which a layer of boundary nodes, $\partial V$, is located in the middle; and a second free space simulation without the boundary layer. In both simulations the sound source $\mathbf{x}_{s}$ emits an acoustic impulse given by Eq. (17).

In the first simulation, sound pressure signals are measured in all the receiver's positions, $\mathbf{x}_{\tau_{\xi}}, \xi=1,2,3 \ldots$ This signals contain not only the direct sound, but also the sound reflected from the boundary, $\partial V$. In the second simulation (in free space), sound pressure signals are measured both at $\mathbf{x}_{\tau_{\xi}}$ and at $\mathbf{x}_{\tau_{\xi}^{\prime}}$. The reason for performing these two simulations is to eliminate numerically the effect of the direct sound in the acoustic response, thus considering only the sound reflected at the boundary $[39,40]$.

In order to fit the reflection factor, the resulting frequency responses obtained from the first simulation (once the direct sound is eliminated) at the receivers, $\mathbf{x}_{\tau_{\xi}}$, are compared to the reference signals obtained from the unbounded simulation at the mirror locations $\mathbf{x}_{\tau_{\xi}^{\prime}}$. To sum up: for each receiver $\mathbf{x}_{\tau_{\xi}}$ we get a value of the reflection factor by comparing the spectra of the signals measured at the receiver location $\mathbf{x}_{\tau_{\xi}}$ and at the mirror location $\mathbf{x}_{\tau_{\xi}^{\prime}}$. Due to the fact that different receivers correspond to different angles of incidence, we can compute in a single numerical experiment, the absolute error, Eq. (18), for any frequency and angle, just by comparing the numerical reflection factor with the theoretical predictions. Finally, all measured signals were windowed with the use of the right half of the Hann window before applying Fourier transforms, in order to reduce signal truncation effects [41].

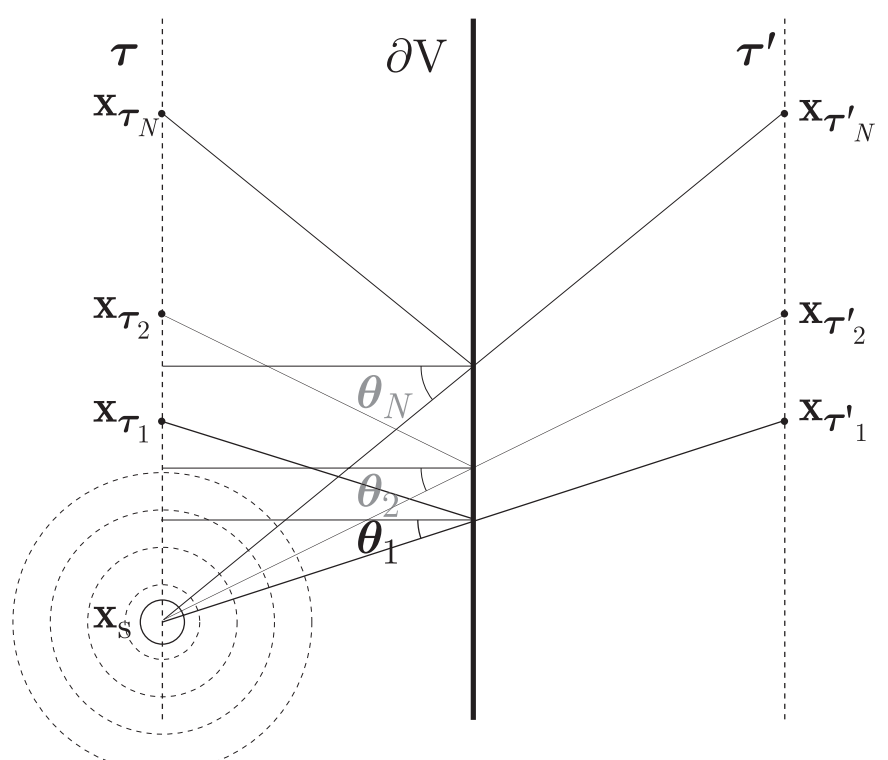

Fig. 4. An ilustrative representation of the experimental setup: The source is located at $\mathbf{x}_{s}$, the receivers are situated over the lines $\tau$ and $\tau^{\prime}$, finally, $\partial V$ represents the boundary layer which is at the same distance from $\mathbf{x}_{\tau}$ to $\mathbf{x}_{\tau^{\prime}}$.

In this particular experiment, a two dimensional domain $V$ of $2000 \times 2000$ nodes is generated with a boundary layer located at the nodes $(i, 1000)$. In this case the sound source is located at node $(520,900)$. The delta Dirac function is approximated again by the same function of Sec. 3 (Eq. (17)) where the time discretization is also fixed at $1 / 16000 \mathrm{~s}$. We fix the space discretization by using $\Delta=\pi c \Delta t / \sqrt{2}$ (see Eq. (5)). The distance between the source and the receiver is varied between 0 and 800 spatial sampling intervals, corresponding to the incident angle $\theta \in\left[0,80^{\circ}\right]$, approximately. It is true that angle values are not distributed homogeneously under this experiment; however, for illustrative purposes, unknown angles are linearly 
interpolated. Finally, the simulation is run for 1024 time steps in order to avoid the numerical rebounds which generate the exterior boundaries and which may produce some disturbances in the analysis of the spectra.

\subsection{Results}

In what follows we will show and analyze the results obtained within the experimental setup defined in the previous section. The results confirm the suitability of the boundary conditions given in Eq. (16) for room acoustics applications. In order to give a complete overview of the performance of the method proposed, we have performed many simulations with different impedances. For clarity, we have used the reflection factor measured in the normal direction, $R_{n}$, as a parameter for the simulations.

The results of the numerical experiments are illustrated in Fig. 5. Each plot corresponds to the absolute error given by Eq. (18) as a function of the angle of incidence, $\theta$, and the frequency. The error is plotted in a graded scale where black corresponds to errors of a few negative $\mathrm{dBs}$ whereas white corresponds to errors less than $-40 \mathrm{~dB}$. The label of each plot is the reflection factor for normal incidence, $R_{n}$, which fixes the value of the impedance $Z$ in the numerical simulation. Therefore for each value of $R_{n}$ (i.e. of $Z$ ) the numerical experiment defined in Sec. 4.1 is performed and the absolute error is computed for different angles given by the receiver locations, $\mathbf{x}_{\tau_{\xi}}$, for all frequencies less than $2500 \mathrm{~Hz}$.

Overall, we obtained low errors (Eq. (18)) in the whole range of impedances and angles. The numerical simulations fit fairly good $(\epsilon \leq-25 \mathrm{~dB})$ from $R_{n}=-1$ to $R_{n}=-0.3$, for all frequencies and angles of incidence. From $R_{n}=-0.2$ to $R_{n}=$ 0.2 , the absolute error increases in the region of high frequencies and small angles of incidence. From $R_{n}=0.2$ to $R_{n}=$ 0.8 the results are again more than acceptable with errors $\epsilon \leq$ $-20 \mathrm{~dB}$. Finally, for $R_{n}=0.9$ and $R_{n}=1$, the absolute error is homogeneous with errors of the order of $-15 \mathrm{~dB}$; however, the limitation to obtain completely reflective surfaces is not new, since it has been also reported in other publications such as Ref. [42].

These results are not unexpected: on the one hand, the regions with values of the absolute error greater than $-10 \mathrm{~dB}$ coincide with those regions in which the theoretic reflection coefficient, $R_{\mathrm{th}}$, is smaller than $-35 \mathrm{~dB}$ when considered in logarithmic scale (see fig. 6). These are quasi-perfect absorbing regions that can not be easily achieved for any multidimensional numerical method (as it is well reported in the technical literature [20]); on the other hand, the increase of error in the range of $R_{n}>0.8$ suggests that the use of a finite difference boundary condition FDO2 with a PSTD approximation for the propagation equation gives an inherent error which gets unacceptable only for $R_{n} \rightarrow$ 1.

It is worth mentioning that highly absorbing materials are unusual in most real scenarios. Therefore, the results are most than acceptable for many purposes such us room acoustics or aeroacoustics.
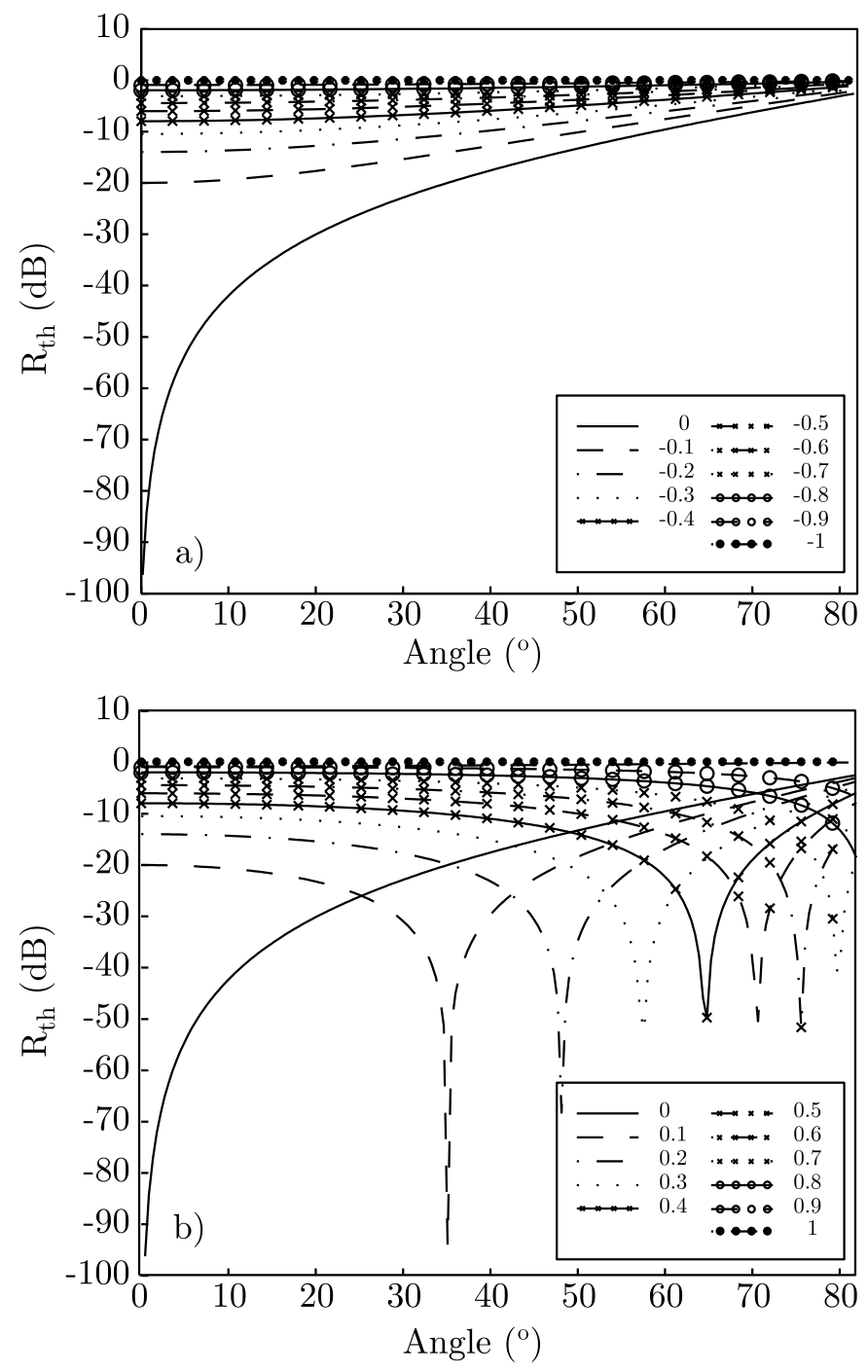

Fig. 6. Different representations of Eq. (9) for different impedances fixed by $R_{n}$. On the top it is plotted $R_{n}$ from -1 to 0 and at the bottom from 0 to 1 .

\section{Conclusions}

Pseudo-spectral time-domain (PSTD) techniques based on Fourier transforms are used nowadays in many different fields such as the propagation of electromagnetic waves [18] or the simulation of photonic devices [24]. In all the cases reported in the literature, PSTD is combined with Perfectly Matched Layer [30] absorbing boundary conditions. In order to use these PSTD methods in room acoustics we developed and validated a numerical implementation of frequency independent impedance boundary conditions.

In this paper we have reviewed and verified the advantages of PSTD methods with respect to the commonly accepted FDTD methods: basically, PSTD displays isotropic propagation and it is more efficient than the usual FDTD methods from a computational point of view. Therefore, PSTD methods seem to be the appropriate simulation schemes for sound propagation within large spaces and for the simulation of long acoustic impulse responses [22]. 

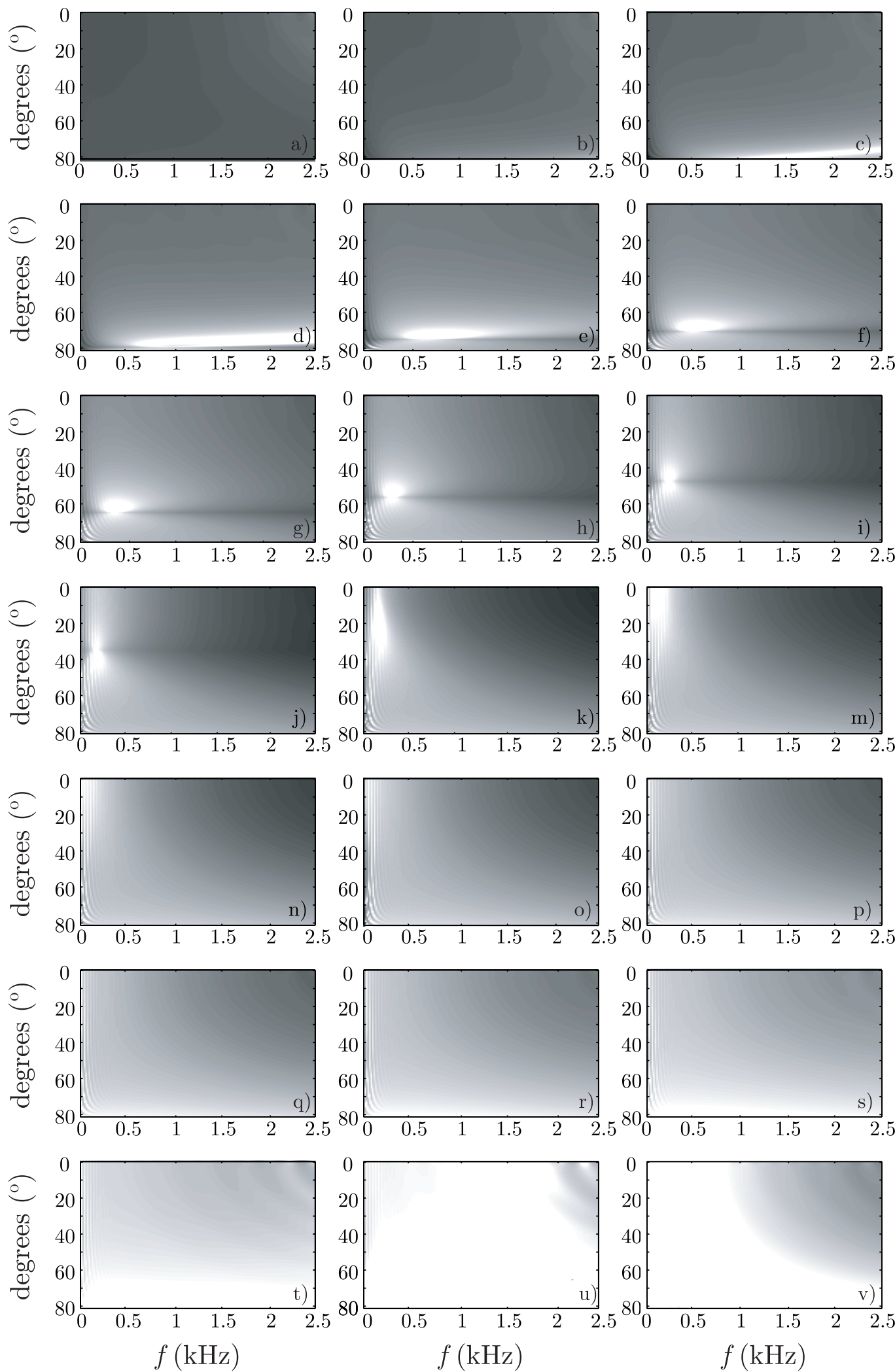

Fig. 5. The absolute error in decibels for different values of the normal reflection coefficient $R_{n}$ obtained with the numerical FDO2 scheme, Eq. (16). From up to down and left to right: a) $R_{n}=1$, b) $R_{n}=0.9$, c) $R_{n}=0.8$, d) $R_{n}=0.7$, e) $\left.R_{n}=0.6, \mathrm{f}\right) R_{n}=0.5$, g) $R_{n}=0.4$, h) $R_{n}=0.3$, i) $R_{n}=0.2$, j) $\left.\left.\left.R_{n}=0.1, \mathrm{k}\right) R_{n}=0, \mathrm{~m}\right) R_{n}=-0.1, \mathrm{n}\right) R_{n}=-0.2$, o) $R_{n}=-0.3$, p) $R_{n}=-0.4$, q) $\left.R_{n}=-0.5, \mathrm{r}\right) R_{n}=-0.6$, s) $R_{n}=-0.7$, t) $\left.R_{n}=-0.8, \mathrm{u}\right)$ $R_{n}=-0.9$ and v) $R_{n}=-1$. 
We have proposed several alternatives in order to simulate locally reacting impedance boundary conditions. Among the proposed boundary schemes we found that the conditions labeled as FDO2 are the most stable and accurate. The FDO2 is defined by Eq. (16) which is the numerical finite-difference approach of the second order impedance boundary condition, Eq. (13). In this paper we show the convenience of combining Eq. (16) for the locally reacting boundary condition with the PSTD scheme for the propagation, Eq. (4).

We performed numerical experiments in order to test the locally reacting behavior of the proposed boundary conditions obtaining fairly good results. In these experiments we computed the absolute error between the numerical and the theoretical reflection factors, analyzing the dependence with frequency and angle of incidence. The absolute error obtained in most cases is below $-25 \mathrm{~dB}$ demonstrating the suitability of the method for the computation of acoustic impulse responses. To sum up: we developed and tested a new impedance boundary conditions for PSTD schemes. In the near future, it would be very interesting to test these boundary conditions by using experimental data.

\section{Acknowledgments}

The work of J. Escolano has been supported by Spanish Ministry of Science and Technology (MCYT) under Project Ref. TEC2006-13883-C04-01 and FEDER funds.

\section{References}

[1] Kutruff H. Room Acoustics. Spon Press, 4th edition; 2000

[2] Schroeder MR. Computer models for concert hall acoustics. Am. J. Phys. 1973; 41:461-471.

[3] Savioja L. Modeling Techniques for Virtual Acoustics. PhD Thesis, Helsinki University of Technology, Telecommunications Software and Multimedia Laboratory, 1999.

[4] Krokstad A., Strom S, and Sorsdal S. Calculating the acoustical room response by the use of a ray tracing technique. J. Sound Vibration 1968; 8:118-125.

[5] Allen JB, and Berkley DA. Image method for efficiently simulating small-room acoustics. J. Acoust. Soc. Am. 1979; 65:943-950.

[6] Funkhouser T, Tsingos N, Carlbom I, Elko G, Sondhi M, West J, Pingali G, Min P, and Ngan A. A beam tracing method for interactive architectural acoustics. J. Acoust. Soc. Am. 2004; 115:739-756.

[7] Wright JR. An Exact Model of Acoustic Radiation in Enclosed Spaces. J. Audio Eng. Soc. 1995; 43:813-820.

[8] Savioja L, Järvinen A, Melkas K, and Saarinen K. Determination of the low frequency behaviour of an IEC listening room. Proc. of the Nordic Acoustical Meeting, 1996; 55-58.

[9] Ciskowski RD, and Brebbia CA. Boundary element methods in acoustics. Computational Mechanics Publications Southampton Boston Co-published with Elsevier Applied Science; 1991.

[10] Botteldooren D. Finite-difference time-domain simulation of lowfrequency room acoustic problems. J. Acoust. Soc. Am. 1995; 98:33023308.

[11] Murphy DT, Kelloniemi A, Mullen J, and Shelley S. Acoustic Modeling using the Digital Waveguide Mesh. IEEE Signal Processing Magazine 2007; 24:55-66.

[12] Hargreaves JA, and Cox TJ.A transient boundary element method model of Schroeder diffuser scattering using well mouth impedance. J. Acoust. Soc. Am. 2008; 124(5):2942-2951.
[13] Savioja L, and Välimäki V. Interpolated rectangular 3-D digital waveguide mesh algorithms with frequency warping. IEEE Trans. on Speech and Audio Processing 2003; 11:783-790.

[14] Fontana F, and Rocchesso D. Physical modeling of membranes for percussion instruments. Acta Acustica United with Acustica 1998; 83:529-542.

[15] Van Duyne SA, and Smith JO. The tetrahedral digital waveguide mesh. Proc. of the IEEE Workshop on Applications of Signal Processing of Audio and Acoustics 1995; 234-237. New Platz, NY.

[16] Miklavcic S, and Ericsson J. Practical implementation of the 3D tetrahedral TLM method and visualization of room acoustics. Int. Conference on Digital Audio Effects (DAFx04) 2004; 262-267. Verona, Italy.

[17] Petrausch S, and Rabenstein R. Simulation of Room Acoustics via Block-Based Physical Modeling with the Functional Transformation Method. IEEE Workshop on Applications of Signal Processing to Audio and Acoustics (WASPAA'05) 2005; 195-198. New Platz, NY.

[18] Liu QH. The PSTD algorithms: a time-domain method requiring only two cells per wavelenght. Microwave and Optical Technology Letters 1997; 15:158-165.

[19] Cooley JW, and Tukey JW. An algorithm for the machine calculation of complex Fourier series. Math. Comput. 1965; 19:297301.

[20] Taflove A, and Hagness SC. Computational Electrodynamics: The Finite-Difference Time-Domain Method. Artech House Publishers; 2000

[21] Spa C., Mateos T, and Garriga A. Methodology for studying the numerical speed of sound in finite differences schemes. Acta Acustica United with Acustica 2009; 95(4):690-695.

[22] Liu QH. The Pseudospectral Time-Domain (PSTD) Algorithm for Acoustic Waves in Absorptive Media. IEEE transactions on ultrasonics, ferroelectrics, and frequency control 1998; 45(4):1044-1055.

[23] Filoux E, Call S, Certon D, Lethiecq M, and Levassort F. Modeling of piezoelectric transducers with combined pseudospectral and finitedifference methods. J. Acoust. Soc. Am. 2008; 123(6):4165-4173.

[24] Pernice WHP. Pseudo-spectral time-domain simulation of the transmission and the group delay of photonic devices. Opt. Quant. Electron 2008; 40:112.

[25] Li Q, Chen Y, and Ge D. Comparison Study of the PSTD and FDTD Methods for Scattering Analysis. Microwave and Optical Technology Letters 2000; 25(3):220-226.

[26] Hoffman JD. Numerical Methods for Engineers and Scientists. MacGraw-Hill, 1992.

[27] Yee KS. Numerical solution of initial boundary values Problems involving Maxwell equations in isotropic media. IEE Transactions on antennas and propagation 1966; 14:302-307.

[28] Wagner C, and Schneider J. An acoustic finite-difference time-domain algorithm with isotropic dispersion. Journal of Computational Acoustics 2005; 13(2):365-384

[29] Proakis JG, and Manolakis DK. Digital Signal Processing: principles, algorithms, and applications. Prentice-Hall, Inc. Upper Saddle River, NJ, USA, 1996.

[30] Bérenger JP. A perfectly matched layer for the absorption of electromagnetic waves. J. Comput. Phys. 1994; 114 (2):185-200.

[31] Bérenger JP. Three-Dimensional Perfectly Matched Layer for the Absorption of Electromagnetic Waves. J. Comput. Phys. 1996; 127 (2):363-379.

[32] Liu QH. PML and PSTD Algorithm for Arbitrary Lossy Anisotropic Media. IEEE Microwave and Guided Wave Letters 1999; 9 (2):48-50.

[33] Hornikx M, and Waxler R. An eigenfunction expansion method to efficiently evaluate spatial derivatives for media with discontinuous properties. Acoustics '08 conference, 29 June - 4 July, Paris, France, 2008.

[34] Fornberg B. A Practical Guide to Pseudospectral Methods. Cambridge University Press, Cambridge, UK, 1996.

[35] Morse PM, and Ingard KU. Theoretical Acoustics. Princeton University Press, 1986.

[36] Strikwerda JC. Finite Difference Schemes and Partial Differential Equations. SIAM, 2004. 
[37] Fan YJ, Ooi BL, and Leong MS. A novel hybrid TDFEM-PSTD method with stability consideration. Microwave and Optical Letters 2005; 47(2):195-197.

[38] Rienstra SW. 1D reflection at an impedance wall. J. Sound Vib. 1975; 125:43-51.

[39] Kelloniemi A, Savioja L, and Välimäki V. Spatial Filter-Based Absorbing Boundary for the 2-D Digital Waveguide Mesh. IEEE Signal Processing Letters 2005; 12(2):126-129.

[40] Kelloniemi A. Improved Adjustable Boundary Condition for the 2-D Digital Waveguide Mesh. Proc. of the 8th Int. Conference on Digital Audio Effects (DAFx 05), Madrid 2005.

[41] Oppenheim AV, Schafer RW and Buck JA. Discrete-time signal processing. Prentice Hall, Upper Saddle River, N.J, 1999.

[42] Huang $X$ and Zhang $X$. A Fourier pseudospectral method for some computational aeroacoustics problems. International Journal of Aeroacoustics 2006; 5 (3):279-294.

\section{Appendix}

In this Appendix, the stability of the proposed boundary conditions is computed through a Von Neumann analysis. For all cases, it is assumed a numerical plane wave of the form $p(\mathbf{x}, t)=\xi^{t / \Delta t} e^{-\iota \mathbf{k}_{0}^{T} \mathbf{x}}$, with a wavenumber $\mathbf{k}_{0}$. The scope is to find the range of values for $Z$ which verify the condition $\|\xi\| \leq 1$ (or alternatively, $\|\xi\|^{2} \leq 1$ ) [36].

\section{FDO1 Stability}

In this section we analyze the stability of the FDO1 boundary condition. After applying the Von Neumann procedure to Eq. (11), we get:

$$
\begin{aligned}
\xi^{n+1} e^{-\iota\left(k_{x_{0}} i \Delta+k_{y_{0}} j \Delta\right)} & =\xi^{n} e^{-\iota\left(k_{x_{0}} i \Delta+k_{y_{0}} j \Delta\right)} \\
& -\frac{Z \Delta t}{\rho \Delta} \xi^{n} e^{-\iota\left(k_{x_{0}} i \Delta+k_{y_{0}} j \Delta\right)} \\
& \left.-\frac{Z \Delta t}{\rho \Delta} \xi^{n} e^{-\iota\left(k_{x_{0}}(i-1) \Delta+k_{y_{0}} j \Delta\right)}\right),
\end{aligned}
$$

where $k_{x_{0}}=\left\|\mathbf{k}_{0}\right\| \cos \theta$ and $k_{y_{0}}=\left\|\mathbf{k}_{0}\right\| \sin \theta$. After simplifying with the factor $\xi^{n} e^{-\iota\left(k_{x_{0}} i \Delta+k_{y_{0}} j \Delta\right)}$ we obtain:

$$
\xi=1-\frac{Z \Delta t}{\rho \Delta}\left(1-e^{\iota k_{x_{0}} \Delta}\right) .
$$

Therefore, the modulus $\|\xi\|^{2}$ is given by,

$$
\begin{aligned}
\|\xi\|^{2} & =\left(1-\frac{Z \Delta t}{\rho \Delta}\left(1-\cos \left(k_{x_{0}} \Delta\right)\right)\right)^{2}+\left(\frac{Z \Delta t}{\rho \Delta} \sin \left(k_{x_{0}} \Delta\right)\right)^{2} \\
& =1+2\left(\left(\frac{Z \Delta t}{\rho \Delta}\right)^{2}+\frac{Z \Delta t}{\rho \Delta}\right)\left(1-\cos \left(k_{x_{0}} \Delta\right)\right) \\
& \leq 1+4\left(\left(\frac{Z \Delta t}{\rho \Delta}\right)^{2}+\frac{Z \Delta t}{\rho \Delta}\right) .
\end{aligned}
$$

Since the stability of the numerical scheme is guaranteed when $\|\xi\|^{2} \leq 1$, the range of allowed values for $Z$ is:

$$
\frac{Z \Delta t}{\rho \Delta}+1 \leq 0
$$

Then, this scheme is stable only if $Z \leq \rho c S^{-1}$, where $S=$ $c \Delta t / \Delta$.

\section{FDO2 Stability}

Following the same procedure, the stability of Eq. (13) leads to;

$\xi=\frac{2 \rho \Delta}{\rho \Delta+0.5 Z \Delta t}-\frac{\rho \Delta-0.5 Z \Delta t}{\rho \Delta+0.5 Z \Delta t} \xi^{-1}+\frac{0.5 \Delta t Z}{\rho \Delta+0.5 Z \Delta t}\left(\xi-\xi^{-1}\right) e^{\iota k_{x_{0}} \Delta}$

which could be seen as a second order polynomial. The solutions for $\xi$ are given by:

$$
\xi=\left\{1,1-\frac{2 Z S /(\rho c)}{1+Z S /(\rho c) \pm \iota \cot \left(k_{x_{0}} \Delta / 2\right)}\right\} .
$$

Although there exists the solution $\xi=1$, one should focus on the more restrictive solution. The next step is to obtain $\|\xi\|$, which is given by,

$$
\|\xi\|=1-\frac{4 Z S /(\rho c)}{\left(1+\frac{Z S}{\rho c}\right)^{2}+\cot ^{2}\left(k_{x_{0}} \Delta / 2\right)} .
$$

Since $\cot (x) \in]-\infty, \infty[$ and it is situated in the denominator, it maximizes the fraction when $\cot ^{2}\left(k_{x_{0}} \Delta / 2\right)=0$, leading to an expression which is always smaller or equal than the unity. Therefore, this scheme is unconditionally stable.

\section{PSO1 Stability}

The application of the Von Neumann analysis to a PSTDbased equation requires to take some care with the spatial derivatives. This section deals with the stability of Eq. (12). For simplicity we derive the stability condition for continuous Fourier transforms. The result of this section can be easily extended to the case of discrete Fourier transform (which is the Fourier transform considered in the simulations presented in the paper) obtaining the same result.

After applying $p(\mathbf{x}, t)=\xi^{t / \Delta t} e^{-\iota \mathbf{k}_{0}^{T} \mathbf{x}}$ on Eq. (12), we obtain the following relation:

$$
\begin{aligned}
\xi^{n+1} & e^{-\iota\left(k_{x_{0}} i \Delta+k_{y_{0}} j \Delta\right)}=\xi^{n} e^{-\iota\left(k_{x_{0}} i \Delta+k_{y_{0}} j \Delta\right)} \\
& -\frac{Z \Delta t}{\rho} \mathcal{F}_{x}^{-1}\left\{j k_{x} \mathcal{F}_{x}\left\{\xi^{n} e^{-\iota\left(k_{x_{0}} i \Delta+k_{y_{0}} j \Delta\right)}\right\}\right\} \\
= & \xi^{n} e^{-\iota\left(k_{x_{0}} i \Delta+k_{y_{0}} j \Delta\right)} \\
- & \frac{Z \Delta t}{\rho} \xi^{n} e^{-\iota k_{y_{0}} j \Delta} \mathcal{F}_{x}^{-1}\left\{j k_{x} \mathcal{F}_{x}\left\{e^{-\iota k_{x_{0}} i \Delta}\right\}\right\} .
\end{aligned}
$$

From the theory of Fourier transforms we have that,

$$
\begin{aligned}
\mathcal{F}_{x}\left\{e^{-\iota k_{x_{0}} i \Delta}\right\} & =2 \pi \delta\left(k_{x}-k_{x_{0}}\right) \\
\mathcal{F}_{x}^{-1}\left\{\iota k_{x} 2 \pi \delta\left(k_{x}-k_{x_{0}}\right)\right\} & =\frac{\partial\left\{e^{-\iota k_{x_{0}} i \Delta}\right\}}{\partial(i \Delta)} \\
& =-\iota k_{x_{0}} e^{-\iota k_{x_{0}} i \Delta} .
\end{aligned}
$$

Using the above expressions, Eq. (26) becomes

$$
\xi=1+\iota k_{x_{0}} \frac{Z \Delta t}{\rho} .
$$

Therefore, $\|\xi\|^{2}$ is given by, 


$$
\|\xi\|^{2}=1+\left(k_{x_{0}} \frac{Z \Delta t}{\rho}\right)^{2},
$$

which means that this scheme is stable only if $Z$ is purely imaginary.

\section{PSO2 Stability}

The stability of Eq. (14) follows the same steps than the previous scheme. After applying same the algebraic simplifications we get,

$$
\xi=2-\xi^{-1}+\frac{Z \Delta t}{2 \rho} \iota k_{x_{0}}\left(\xi-\xi^{-1}\right) .
$$

Rearranging this equation, we obtain,

$$
\xi^{2}\left(1-\iota k_{x_{0}} \frac{Z \Delta t}{2 \rho}\right)-2 \xi+\left(1+\iota k_{x_{0}} \frac{Z \Delta t}{2 \rho}\right)=0 .
$$

Since Eq. (32) is a second order polynomial, we obtain two solutions for $\xi$ :

$$
(\xi-1)\left(\xi-\frac{1+\iota k_{x_{0}} \frac{Z \Delta t}{2 \rho}}{1-\iota k_{x_{0}} \frac{Z \Delta t}{2 \rho}}\right)=0
$$

The first root $(\xi-1)$ accomplishes with the stability condition. Therefore, the second root has to be analyzed in detail. After calculating the modulus of this root, it is obtained the following expression

$$
\|\xi\|=\left\|\frac{1+\iota k_{x_{0}} \frac{Z \Delta t}{2 \rho}}{1-\iota k_{x_{0}} \frac{Z \Delta t}{2 \rho}}\right\|=1,
$$

which implies that this scheme is unconditionally stable. 